EGU2014-7019

European Geosciences Unio

27 April - 02 May 2014, Vienna, Austria

Introduction

To determine the gravity field of the Moon, the two satellites of the NASA mission GRAIL (Gravity Recovery and Interior Laboratory) were
launched on September 102011 and reached their lunar orbits in the beginning of 2012 (Zuber et al., 2013). The concept of the mission was inherited from the Earth-orbiting mission GRACE (Gravity Recovery and Climate Experiment) in that the key observations consisted of ultra-precise interway Doppler observations from the NASA Deep Space Network (DSN) W an unprecedented accuracy for both the near- and the far-side of the Moon. The first official GRAIL gravity field models contain spherical harLemoine at., 2013).

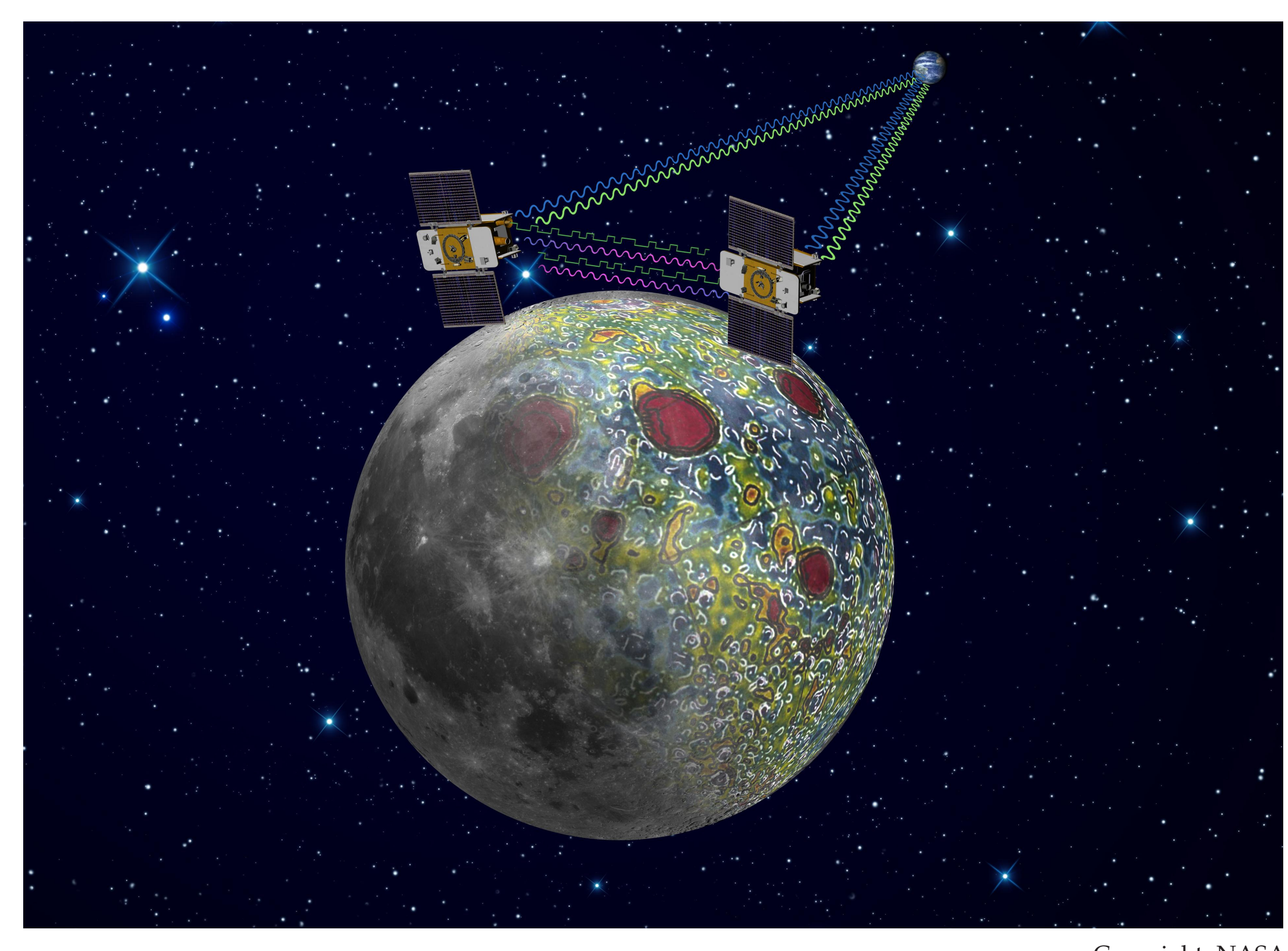

Based on our experience in GRACE data processing, we are adapting our (CMA, Beutler et al., 2010), to the GRAIL mission within the Bernese GNSS absure. We use the Bernese GNSS software is still at the outset - the pre-processed reduceddynamic GNIIB position data as pseudo-observations (relative weighting
$\left.10^{8}: 1\right)$. The following results are based on the release 3 data of the pri-

The Celestial Mechanics Approach (CMA)

The idea of the CMA is to rigorously treat the gravity field recovery as an for appropriately constrined stochastic pulses (instanteproach allowing velocity) to compensate for inevitable model deficiencies. For each satelite, the equations of motion to be solved read as $\ddot{\mathbf{r}}=\mathbf{a}_{G}+\mathbf{a}_{P}$, where
$\mathrm{a}_{C}=\nabla V$ denotes the acceleration due to the gravity potential $V$, which we parametrize in terms of the standard $\mathrm{SH}$ expansion. $\mathrm{a}_{P}$ denotes the ccording to JPL ephemerides DE 421 , forces due to the tidy perturbation of the Moon and relativistic corrections. We do not yet model directo indirect solar radiation pressure. observations contribute to one and the same set of parameters, whic are simultaneously esti

Orbits: Initial conditions every 24h; once-per-revolution accelera-
tions in $\mathrm{R}, \mathrm{S}, \mathrm{W}$ (radial, along-track, out-of-plane); stochastic pulses

- Static gravity
and order 200

- Ka-band: Time bias every $24 \mathrm{~h}$

\title{
GRAIL Gravity Field Determination Using the Celestial Mechanics Approach
}

Orbits

In a first step, we estimate a priori orbits using the GNI1B positions and KBRR abser, whions. Hig. 1 shows that their quarty strongly depends on

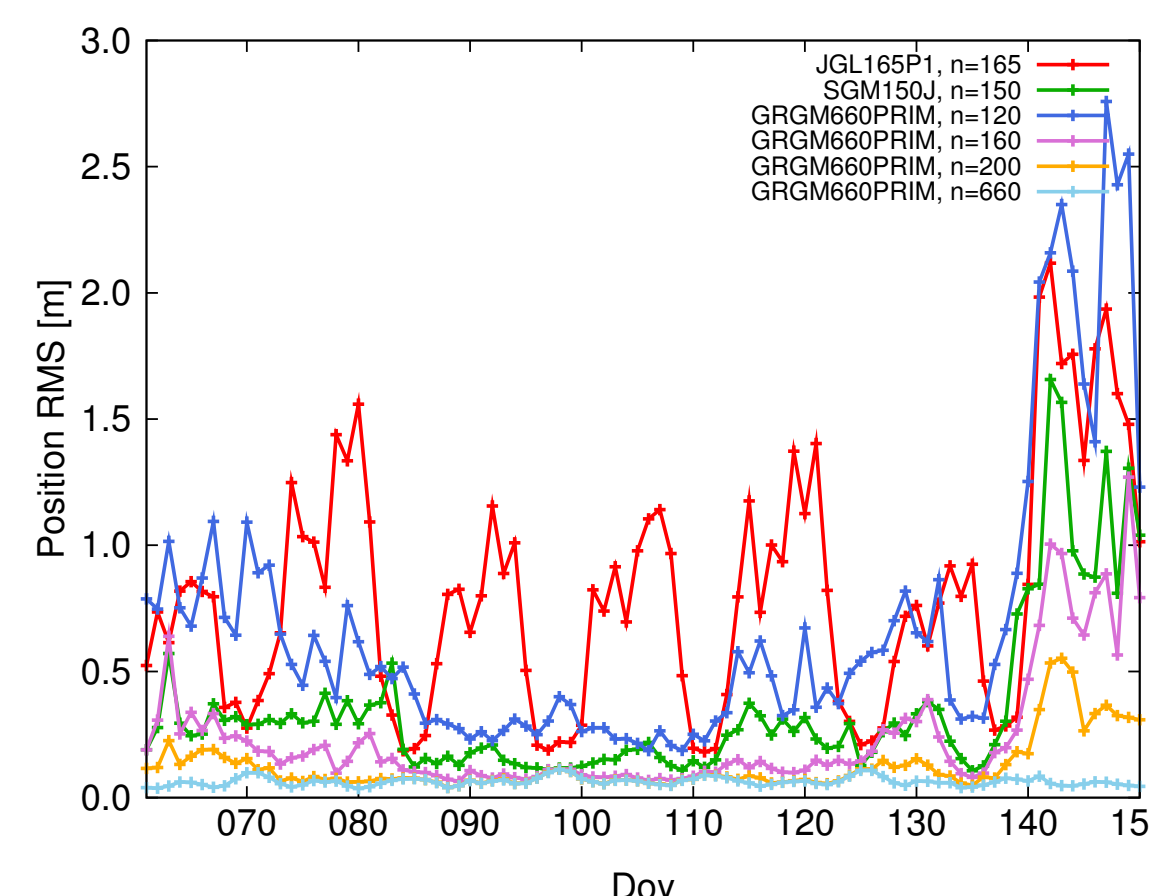

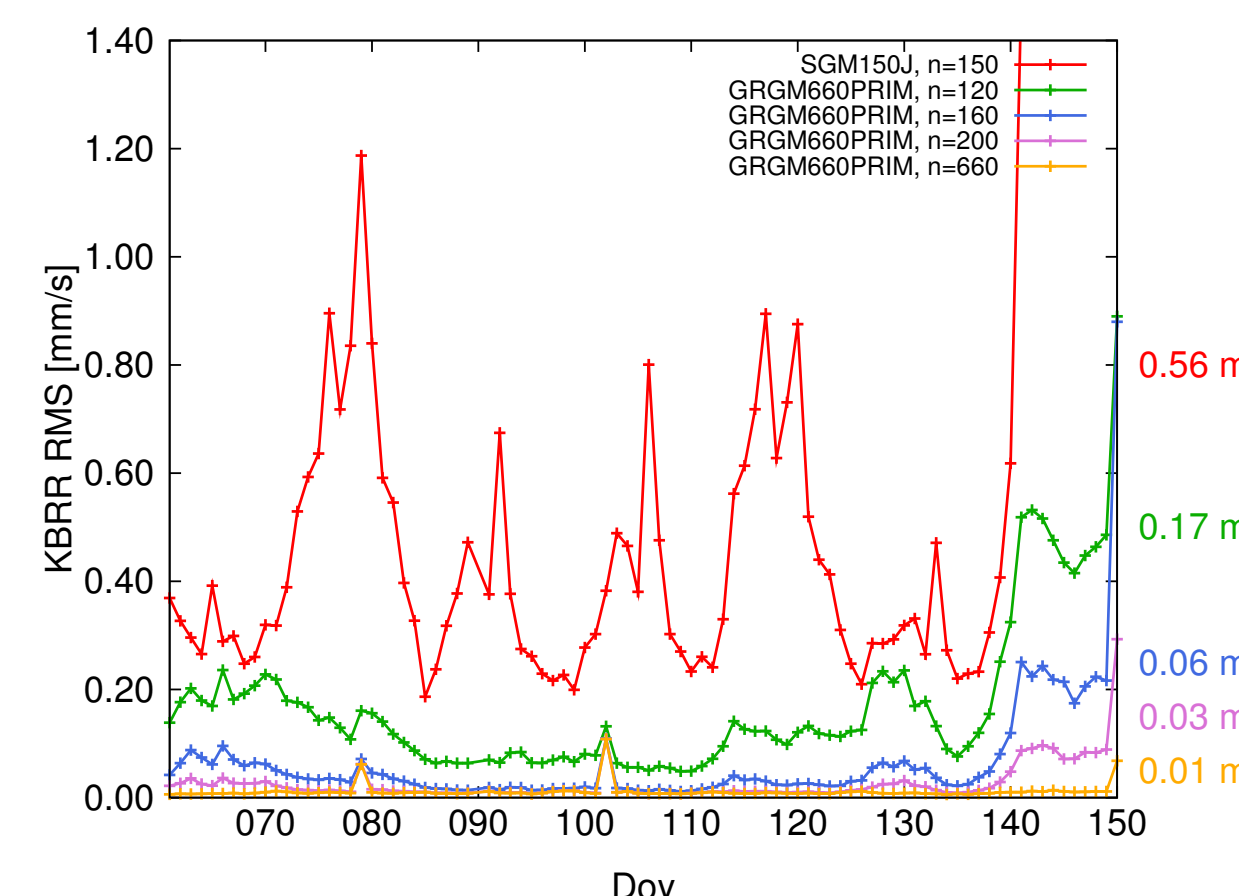

Figure 1: Left: RMS values of the GNII position fit. Right: RMS values of the KBRR residusing the Lunar Prospector (UGL165PI) or SELLNE ( (SGM 150J) gravity field and become be
ter (more consistent) when ter (more consistent) when introducing NASA's first
(Lemoine et al., 2013), truncated at the degrees indicated.

Fig. 2 (left) shows Ka-band residuals for day 069 . The gravity fiel GRGM660PRIM was used up to degree and order 660. Compared to the large and show clear once-per-revolution signals. The green and blue b indicate the time spans during which each satellite is in sunlight The obvious correlation between these time spans and the large residuals suggest that radiation pressure modeling is crucial. The residuals turn out muc larger, if one does not allow for a Kaband time bias, i.e. a shift in obse mission phase this bias is at around one second (see Fig. 2 ng

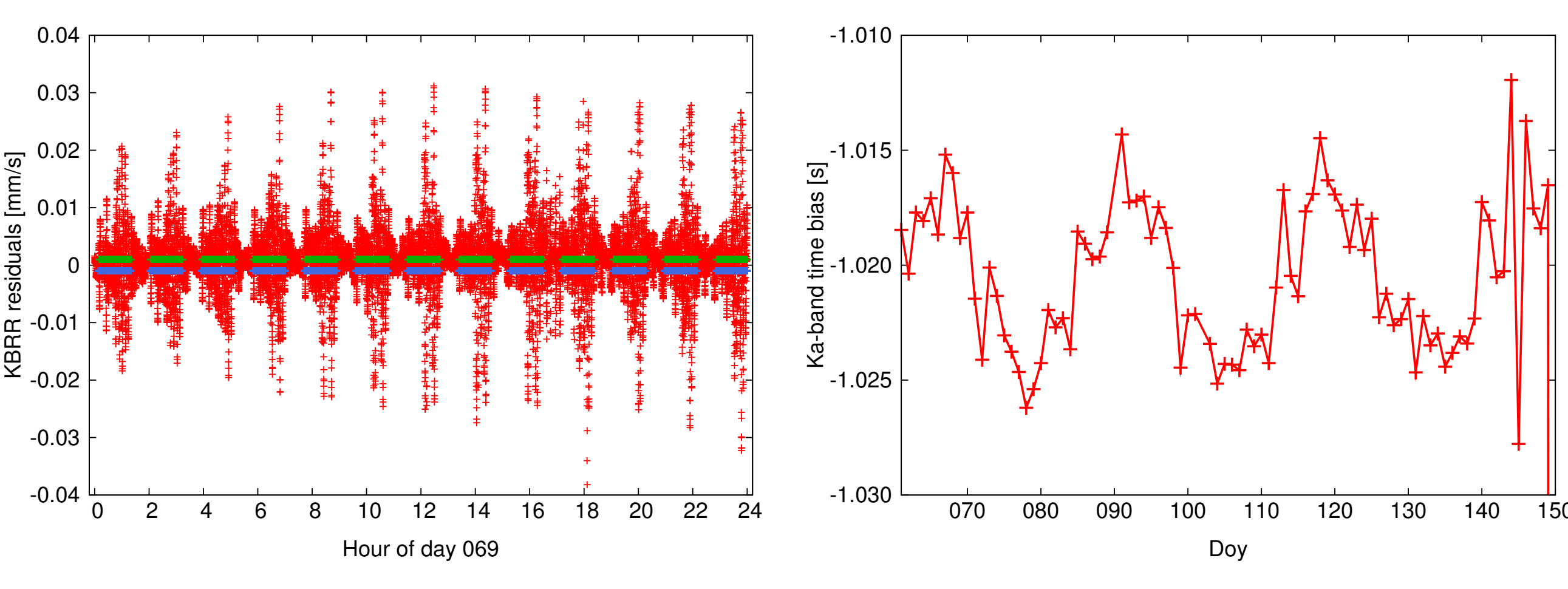

Figure 2: Left: KBRR residuals and time spans for which GRAIL-A (green) and GRAIL-B
(blue) are in s:

\section{Gravity field}

We set up stochastic pulses every 40 minutes. This value is a compromuch of the gravity signal. Fig. 3 (left) shows the difference degree amplitudes (w.r.t. GRGM600PRIM) for different intervals between the stochastic

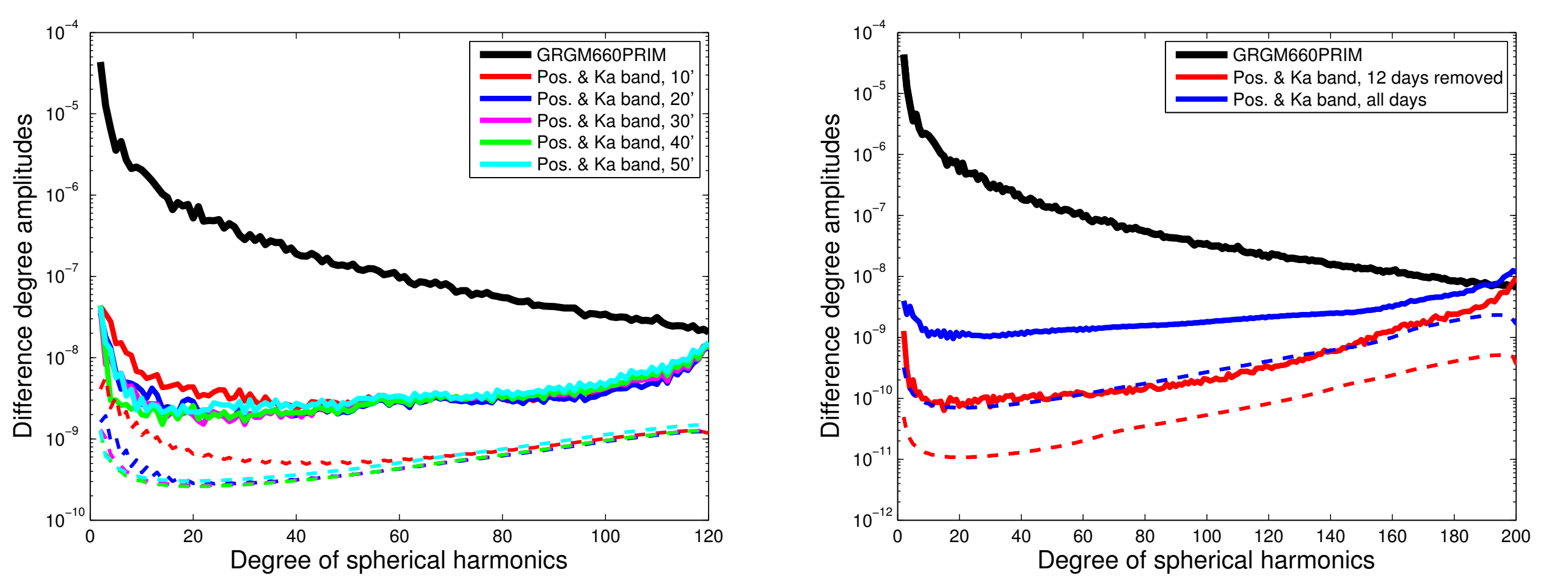

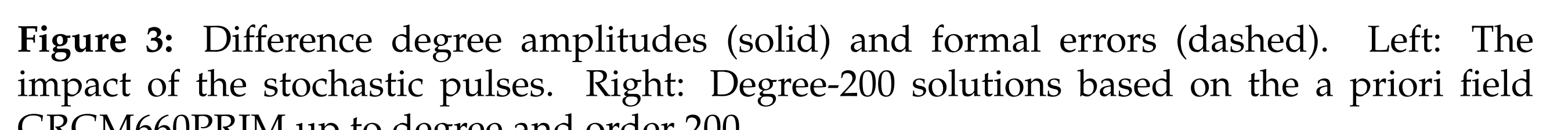

The orbits determined in the first step serve as a priori orbits for a comare stacked to weekly, monthly and finally three-monthly NEQs, which are then inverted. Fig. 3 (right) shows the difference degree amplitudes (200). The (ap problematic days with larger residuns is completely skippen the data of 12 curve). Data and residual screening still have to be refined to keep the maximum amount of data without degrading the solution. Fig. 4 shows
the gravity anomalies of the degree-200 solution.

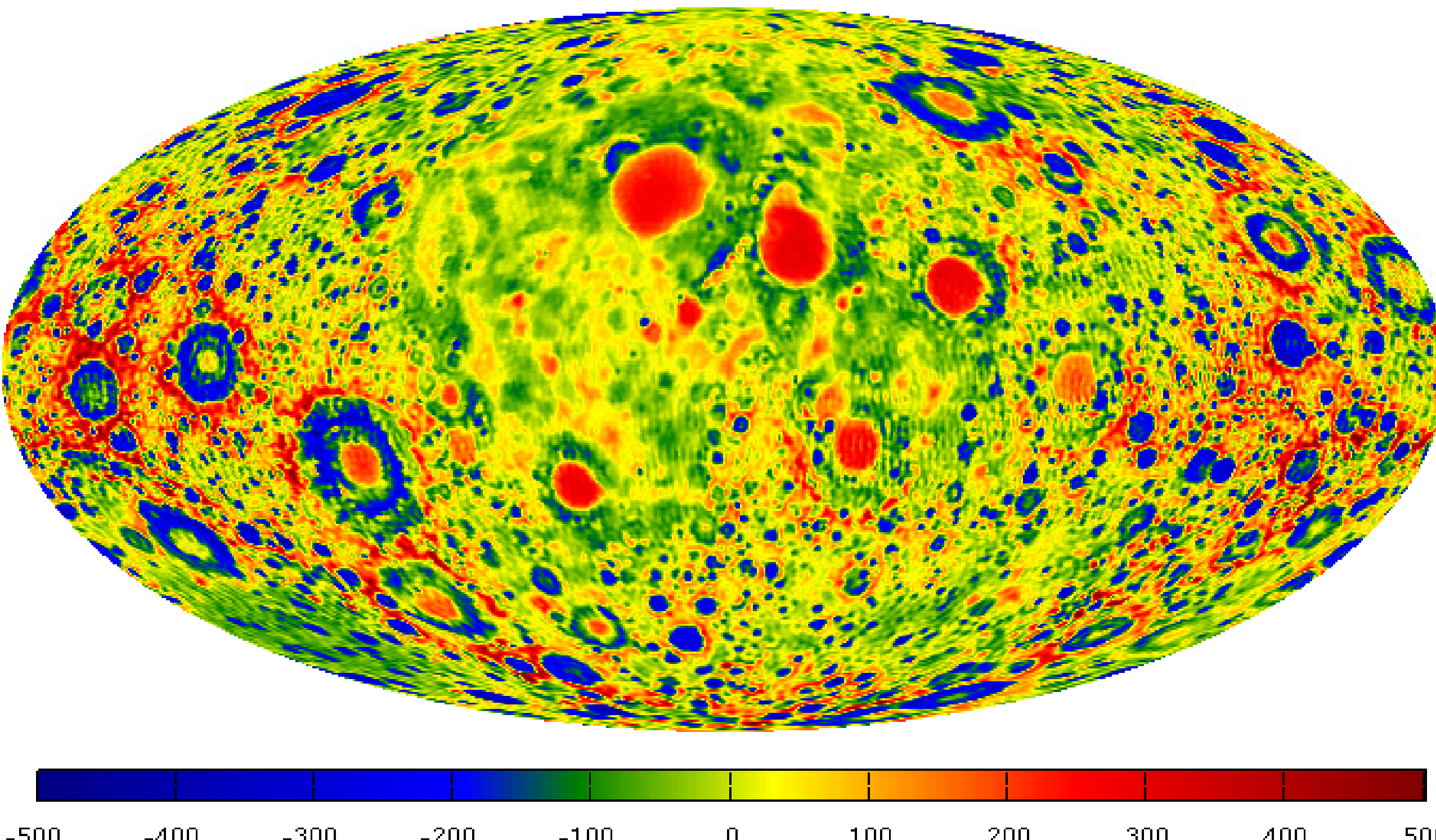

Figure 4: Free-air gravity anomal
tered around meridian (nearside).

An important feature of the ity for the used a priori field. Fig. 5 shows difference degree amplitudes of solutions
obtained with the indicated obtained with the indicated
p priori fields. Already in a priori fields. Already in
the first iteration the SELENE feld is improved basically to the same field as when startdegree and order 120 . The poorer on the far-side) lea to a comparable result after the second iteration.

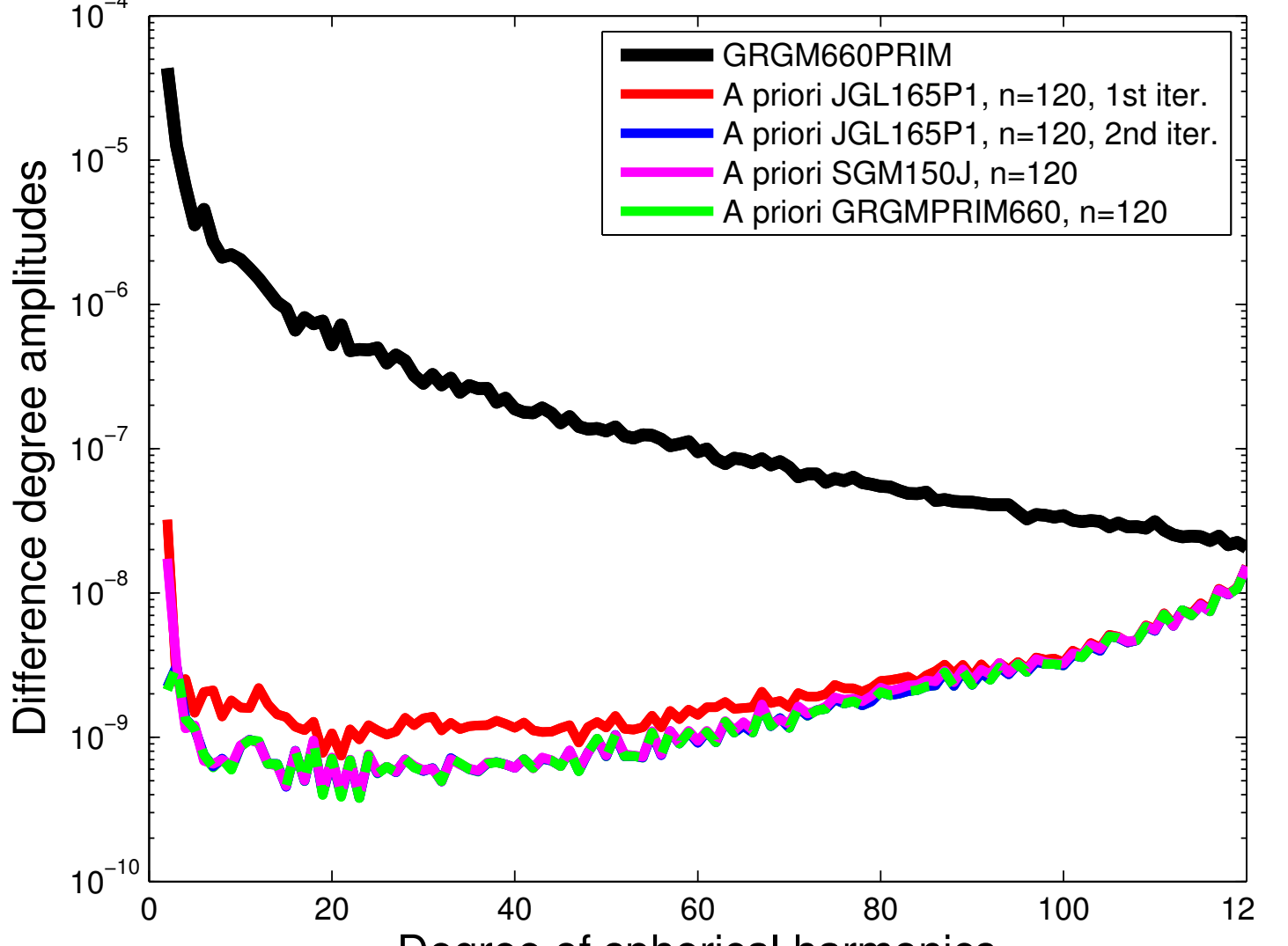

Doppler data processing

Besides the KBRR observations, GRAIL orbit and gravity field determination is based on its Doppler tracking by several Earth-based stations of the
DSN. The basic signal is the frequency registered at the reception station DSN. The basic signal is the frequency registered at the reception station
based on cycles accumulated over a given time. In practice, Doppler observables are reconstructed from the travel time of a series of radio signals

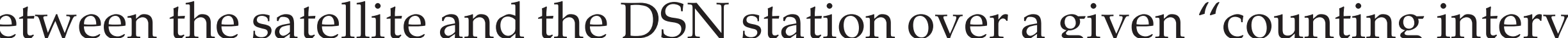
$T_{c}$ (Moyer, 2000) as

$$
\mathcal{D}_{C}=M_{2} f_{T}(t) \frac{\rho_{e}-\rho_{s}}{T_{c}}
$$

where $\mathcal{D}_{C}$ is the computed Doppler $(C), \rho_{s}$ and $\rho_{e}$ are precision light-time ratio (a constant scaling factor applied by the probe to the frequency of the tracking signal) and $f_{T}(t)$ is the transmitter frequency.
D. Arnold, S. Bertone, A. Jäggi, G. Beutler, H. Bock, U. Meyer, L. Mervart Astronomical Institute, University of Bern, Bern, Switzerland

order to process GRAIL Doppler observatos, we then need an andt(n)

- the trajectory of the tracking station and a GRAIL orbit (from a arycentric Celestial Reference System),

- a modeling of biases and non-geometrical effects in the Doppler siges (for relativistic effects).

The difference between the computed Doppler and the observed Doppler normal equation to be solved in the orbit determination process. We are currently improving the Doppler modeling in the Bernese GNSS software $\mathrm{in}$ order to reach the $\mathrm{mm} / \mathrm{s}$ accuracy before undertaking the orbit determi-
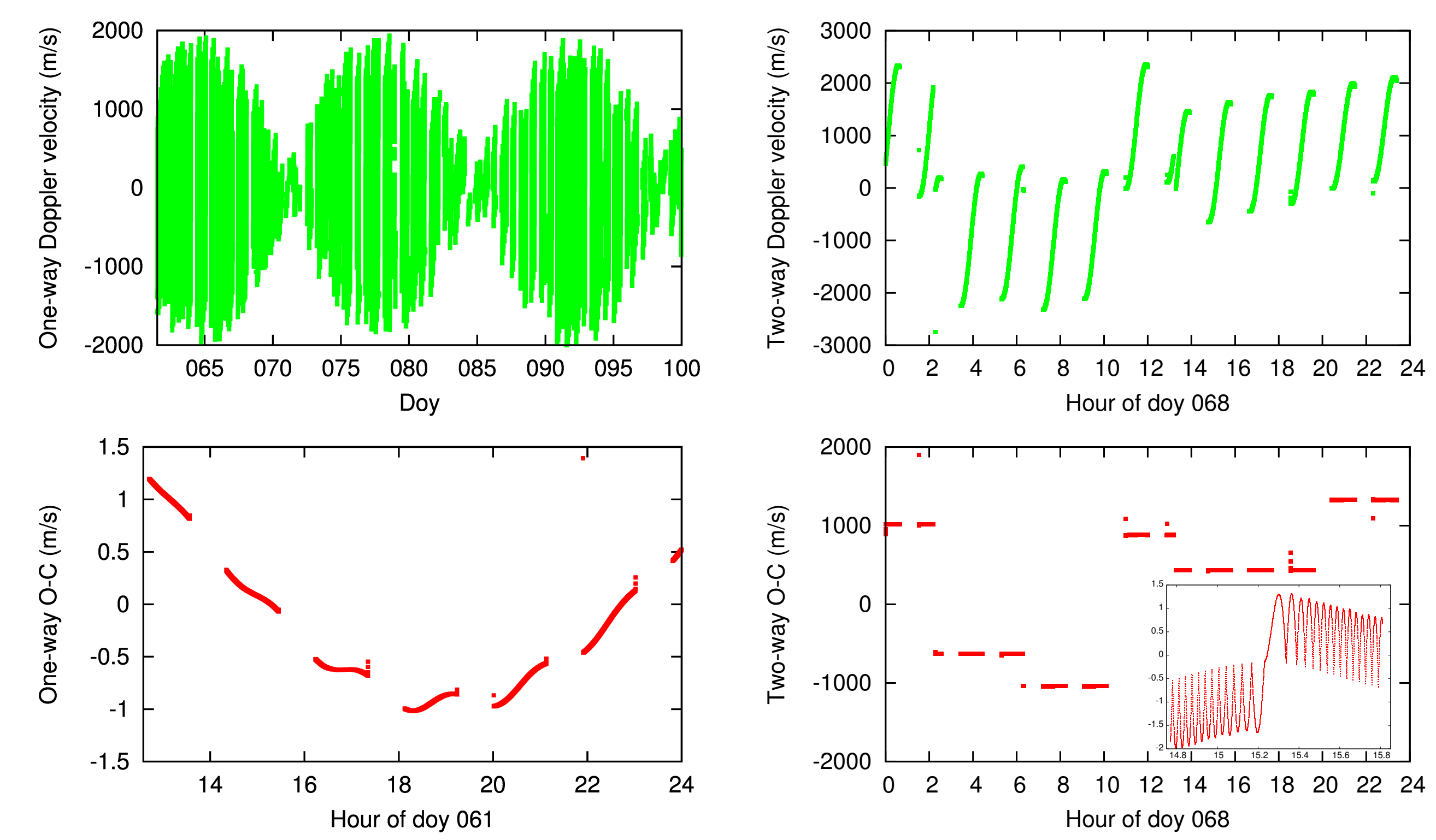

Figure 6: Current status of X-band one-way (left) and S-band two-way (right) Doppler ob-

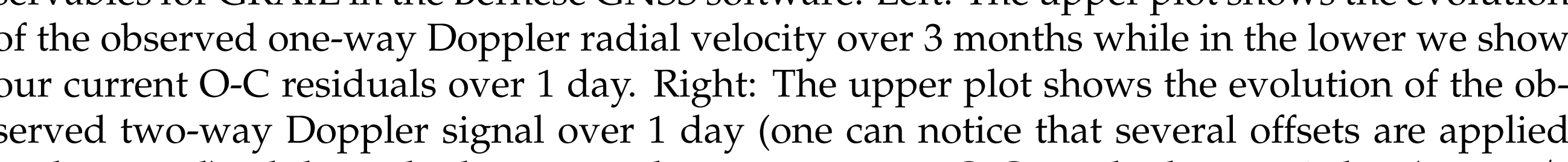
served two-way Doppler signal over 1 day (one can notice that several offsets are applied
to the signal) while in the lower we hsow our current $\mathrm{O}-\mathrm{C}$ residuals over 1 day $(\sim 1 \mathrm{~m} / \mathrm{s}$
amplitude in each offset interval, see zoomed plot)

Conclusions

- The adaption of the CMA from GRACE to GRAIL allows for first Iunar gravity fields obtained with the Bernese GNSS software.

- Further investigations are necessary to fully exploit the precision of the

- We are making progress in the implementation of DSN data analyof the GNIIB products may be used for orbit and gravity field determination, which is necessary to obtain fully independent solutions.

References

Beutler et al. (2010) The celestial mechanics approach: theoretical foundations. IGeod 84:605.
624 and The celestial mechanics approach: application to data of the GRACE mission. J Konopliv vetal. (2013) The JPL lunar gravity field to spherical harmonic degree 660 from the GRAIL Primary Mission.J. Geophys. Res. Planets $118,1415-1434$
Lemoine et al . 2013 ) High-degree gravity model from GRALL primary mission data. J. Geophys. Reses. Planets 1118, $16767-1698$
Mover (2000) Formulation for Observed and Computed Values of Deep Space Network Data Moyer (2000) Formulation for Observed and Computed Values of Deep Space Network Data
Types for Navigation. JPL Publications Types for Navigation. JPL Publications
Zuber ex tal. (2013) Gravity field of the moon from the gravity recovery and interior laboratory

Contact address 